\title{
Speculative trade under unawareness: the infinite case
}

\author{
Martin Meier • Burkhard C. Schipper
}

Received: 26 August 2013 / Accepted: 17 April 2014 / Published online: 30 May 2014

(C) SAET 2014

\begin{abstract}
We prove a "no-speculative-trade" theorem under unawareness for the infinite case. This generalizes the result for the finite case by Heifetz et al. (Games Econ Behav 77:100-121, 2013).
\end{abstract}

Keywords Awareness - Unawareness - Speculation · Trade - Agreement · Common prior $\cdot$ Common certainty

JEL Classification $\quad$ C70 $\cdot$ C72 $\cdot$ D53 $\cdot$ D80 $\cdot$ D82 $\cdot$ D84.

\section{Introduction}

Unawareness refers to the lack of conception rather than to the lack of information. It is natural to presume that asymmetric unawareness may lead to speculative trade. Indeed, Heifetz et al. (2013) present a simple example of speculation under unawareness in which there is common certainty of willingness to trade but agents have a strict preference to trade despite the existence of a common prior. ${ }^{1}$ This is impossible in standard state-space structures with a common prior. In standard "no-speculativetrade" theorems, if there is common certainty of willingness to trade, then agents are necessarily indifferent to trade (Milgrom and Stokey 1982). Somewhat surprisingly, Heifetz et al. (2013) also prove a "no-speculative-trade" result according to which

\footnotetext{
1 This example is a probabilistic version of the speculation example in Heifetz et al. (2006).

M. Meier

Institut für Höhere Studien (IHS), Vienna, Austria

B. C. Schipper $(\varangle)$

Department of Economics, University of California, Davis, USA

e-mail: bcschipper@ucdavis.edu
} 
under a common prior there cannot be common certainty of strict preference to trade. This means that arbitrarily small transaction costs rule out speculation under asymmetric unawareness. The "no-speculative-trade" result in Heifetz et al. (2013) was stated for finite unawareness belief structures. In this note, we generalize the result to infinite unawareness belief structures. Such a generalization is relevant since the space of underlying uncertainties may be large. Especially if it is large, agents may be unaware of some of them. Moreover, the generalization serves as a robustness check for our "no-speculative-trade" result for finite unawareness belief structures. It shows that the result in Heifetz et al. (2013) is not an artifact of the finiteness assumption but holds more generally. The topological unawareness belief structure introduced in this paper to prove our result may be of independent interest for other applications.

Board and Chung (2011) present a different model of unawareness, in which unawareness is about "objects" rather than events. Intuitively, we model an agent's unawareness of events like "penicillium rubens has antibiotic properties" while in their model the agent's corresponding unawareness would be about "penicillium rubens". Board and Chung (2011) also prove a "no-speculative-trade" result for finite spaces. This suggests that the "no-speculative-trade" result in Heifetz et al. (2013) remains robust if unawareness of events is replaced by unawareness of objects. Further, Board and Chung (2011) study awareness of unawareness of objects. This allows them to show that the "no-speculative-trade" result obtains both under "living in denial" and "living under paranoia", where former refers to the situation in which every agent is certain that there is nothing they are unaware of, while latter refers to the situation in which every agent is certain that there is something they are unaware of. Grant and Quiggin (2013) discuss the speculative trade example of Heifetz et al. (2006) in the context of awareness of unawareness. The authors argue that agents should induce from previous experiences of becoming aware and from differences in awareness across agents that they themselves could be unaware of something. This awareness of unawareness may be coupled with a version of a precautionary principle which may make them reluctant to engage in speculative trade.

The paper is organized as follows. The next section introduces topological unawareness belief structures. The general "no-speculative-trade" theorem is stated in Sect. 3. Finally, Sect. 4 contains the proof of the theorem.

\section{Topological unawareness belief structures}

We consider an unawareness belief structure as defined in Heifetz et al. (2013) but with additional topological properties.

\subsection{Compact Hausdorff state-spaces}

Let $\mathcal{S}=\{S\}$ be a complete lattice of disjoint state-spaces, with respect to the partial order $\preceq$ on $\mathcal{S}$. If $S^{\prime}$ and $S^{\prime \prime}$ are such that $S^{\prime \prime} \succeq S^{\prime}$ we say that $S^{\prime \prime}$ is more expressive than $S^{\prime} .(\mathcal{S}, \preceq)$ is well-founded, that is, every nonempty subset $\mathcal{X} \subseteq \mathcal{S}$ contains a $\preceq$-minimal element. That is, there is a $S^{\prime} \in \mathcal{X}$ such that for all $S \in \mathcal{X}:$ if $S \preceq S^{\prime}$, then $\bar{S}=S^{\prime}$. Each state-space $S \in \mathcal{S}$ is a nonempty compact Hausdorff space with a Borel 
$\sigma$-field $\mathcal{F}_{S}$. Denote by $\Omega=\bigcup_{S^{\prime} \in \mathcal{S}} S^{\prime}$ the union of these spaces. $\Omega$ is endowed with the disjoint union topology: $O \subseteq \Omega$ is open if and only if $O \cap S$ is open in $S$ for all $S \in \mathcal{S}$.

As we will see later, the lattice of spaces is useful to model unawareness with multiple agents. If an agent forms beliefs about events of a space with low expressive power and not about some events of a space with higher expressive power, then she is unaware of latter events. In a multi-agent context, agents should also form beliefs about other agent's awareness. In particular, at some events that agent $i$ forms beliefs over, agent $j$ may form beliefs about events in a space with lower expressive power. It means that $i$ thinks that $j$ is unaware of some aspects of the reality. Interactive unawareness is captured by forming beliefs about beliefs etc. down the lattice. We will make this precise once we introduce agents' type mappings in Sect. 2.8.

\subsection{Continuous projections}

For every $S$ and $S^{\prime}$ such that $S^{\prime} \succeq S$, there is a continuous surjective projection $r_{S}^{S^{\prime}}: S^{\prime} \rightarrow S$, where $r_{S}^{S}$ is the identity. Note that the cardinality of $S$ is smaller than or equal to the cardinality of $S^{\prime}$. We require the projections to commute: if $S^{\prime \prime} \succeq S^{\prime} \succeq S$ then $r_{S}^{S^{\prime \prime}}=r_{S}^{S^{\prime}} \circ r_{S^{\prime}}^{S^{\prime \prime}}$. If $\omega \in S^{\prime}$, denote $\omega_{S}=r_{S}^{S^{\prime}}(\omega)$. If $D \subseteq S^{\prime}$, denote $D_{S}=\left\{\omega_{S}: \omega \in D\right\}$.

\subsection{Events}

For $D \subseteq S$, denote $D^{\uparrow}=\bigcup_{S^{\prime} \in \mathcal{S}: S^{\prime} \succeq S}\left(r_{S}^{S^{\prime}}\right)^{-1}(D)$. An event is a pair $(E, S)$, where $E=D^{\uparrow}$ with $D \subseteq S$, where $S \in \mathcal{S}$. D is called the base and $S$ the base-space of $(E, S)$, denoted by $S(E)$. If $E \neq \varnothing$, then $S$ is uniquely determined by $E$ and, abusing notation, we write $E$ for $(E, S)$. Otherwise, we write $\emptyset^{S}$ for $(\emptyset, S)$. Note that not every subset of $\Omega$ is an event.

Let $\Sigma$ be the set of measurable events of $\Omega$, i.e., $D^{\uparrow}$ such that $D \in \mathcal{F}_{S}$, for some state-space $S \in \mathcal{S}$. Note that unless $\mathcal{S}$ is a singleton, $\Sigma$ is not an algebra because it contains distinct $\emptyset^{S}$ for all $S \in \mathcal{S}$.

\subsection{Negation}

If $\left(D^{\uparrow}, S\right)$ is an event where $D \subseteq S$, the negation $\neg\left(D^{\uparrow}, S\right)$ of $\left(D^{\uparrow}, S\right)$ is defined by $\neg\left(D^{\uparrow}, S\right):=\left((S \backslash D)^{\uparrow}, S\right)$. Note, that by this definition, the negation of a (measurable) event is a (measurable) event. Abusing notation, we write $\neg D^{\uparrow}:=(S \backslash D)^{\uparrow}$. Note that by our notational convention, we have $\neg S^{\uparrow}=\emptyset^{S}$ and $\neg \emptyset^{S}=S^{\uparrow}$, for each space $S \in \mathcal{S}$. $\neg D^{\uparrow}$ is typically a proper subset of the complement $\Omega \backslash D^{\uparrow}$. That is, $(S \backslash D)^{\uparrow} \varsubsetneqq \Omega \backslash D^{\uparrow}$.

Intuitively, there may be states in which the description of an event $D^{\uparrow}$ is both expressible and valid-these are the states in $D^{\uparrow}$; there may be states in which its description is expressible but invalid - these are the states in $\neg D^{\uparrow}$; and there may be states in which neither its description nor its negation is expressible. The definition 
of negation is crucial for modeling unawareness in our structures. Intuitively, if an agent considers the negation of an event $E$, then she does not necessarily considers everything but $E$. Rather, she consider everything but $E$ given her awareness level as defined by the space on which her beliefs are defined on.

\subsection{Conjunction and disjunction}

If $\left\{\left(D_{\lambda}^{\uparrow}, S_{\lambda}\right)\right\}_{\lambda \in L}$ is a finite or countable collection of events (with $D_{\lambda} \subseteq S_{\lambda}$, for $\lambda \in L)$, their conjunction $\bigwedge_{\lambda \in L}\left(D_{\lambda}^{\uparrow}, S_{\lambda}\right)$ is defined by $\bigwedge_{\lambda \in L}\left(D_{\lambda}^{\uparrow}, S_{\lambda}\right):=$ $\left(\left(\bigcap_{\lambda \in L} D_{\lambda}^{\uparrow}\right), \sup _{\lambda \in L} S_{\lambda}\right)$. Note, that since $\mathcal{S}$ is a complete lattice, $\sup _{\lambda \in L} S_{\lambda}$ exists. If $S=\sup _{\lambda \in L} S_{\lambda}$, then we have $\left(\bigcap_{\lambda \in L} D_{\lambda}^{\uparrow}\right)=\left(\bigcap_{\lambda \in L}\left(\left(r_{S_{\lambda}}^{S}\right)^{-1}\left(D_{\lambda}\right)\right)\right)^{\uparrow}$. Again, abusing notation, we write $\bigwedge_{\lambda \in L} D_{\lambda}^{\uparrow}:=\bigcap_{\lambda \in L} D_{\lambda}^{\uparrow}$ (we will therefore use the conjunction symbol $\wedge$ and the intersection symbol $\cap$ interchangeably).

We define the relation $\subseteq$ between events $(E, S)$ and $\left(F, S^{\prime}\right)$, by $(E, S) \subseteq\left(F, S^{\prime}\right)$ if and only if $E \subseteq F$ as sets and $S^{\prime} \preceq S$. If $E \neq \emptyset$, we have that $(E, S) \subseteq\left(F, S^{\prime}\right)$ if and only if $E \subseteq F$ as sets. Note however that for $E=\emptyset^{S}$, we have $(E, S) \subseteq\left(F, S^{\prime}\right)$ if and only if $S^{\prime} \preceq S$. Hence, we can write $E \subseteq F$ instead of $(E, S) \subseteq\left(F, S^{\prime}\right)$ as long as we keep in mind that in the case of $E=\emptyset^{S}$ we have $\emptyset^{S} \subseteq F$ if and only if $S \succeq S(F)$. It follows from these definitions that for events $E$ and $F, E \subseteq F$ is equivalent to $\neg F \subseteq \neg E$ only when $E$ and $F$ have the same base, i.e., $S(E)=S(F)$.

The disjunction of $\left\{D_{\lambda}^{\uparrow}\right\}_{\lambda \in L}$ is defined by the de Morgan law $\bigvee_{\lambda \in L} D_{\lambda}^{\uparrow}:=$ $\neg\left(\bigwedge_{\lambda \in L} \neg\left(D_{\lambda}^{\uparrow}\right)\right)$. Typically $\bigvee_{\lambda \in L} D_{\lambda}^{\uparrow} \varsubsetneqq \bigcup_{\lambda \in L} D_{\lambda}^{\uparrow}$, and if all $D_{\lambda}$ are nonempty we have that $\bigvee_{\lambda \in L} D_{\lambda}^{\uparrow}=\bigcup_{\lambda \in L} D_{\lambda}^{\uparrow}$ holds if and only if all the $D_{\lambda}^{\uparrow}$ have the same base-space. Note, that by these definitions, the conjunction and disjunction of (at most countably many measurable) events is a (measurable) event.

Apart from the topological conditions and the well-foundedness assumption, the event-structure outlined so far is analogous to Heifetz et al. (2006, 2008); Heifetz et al. (2013).

\subsection{Regular borel probability measures}

Here and in what follows, the term 'events' always means measurable events in $\Sigma$ unless otherwise stated.

For each $S \in \mathcal{S}, \Delta(S)$ is the set of regular Borel probability measures on $\left(S, \mathcal{F}_{S}\right)$. We consider this set itself as a measurable space which is endowed with the topology of weak convergence. ${ }^{2}$

\footnotetext{
2 The topology of weak convergence is generated by the sub-basis of sets $\{\mu \in \Delta(S): \mu(O)>r\}$ where $O \subseteq S$ is open and $r \in \mathbb{R}$ (see e.g. Billingsley 1968, appendix III). This topology coincides with the weak*
} 
$\bigcup_{S \in \mathcal{S}} \Delta(S)$ is endowed with the disjoint union topology: $O_{\Delta} \subseteq \bigcup_{S \in \mathcal{S}} \Delta(S)$ is open if and only if $O_{\Delta} \cap \Delta(S)$ is open in $\Delta(S)$ for all $S \in \mathcal{S}$.

Note that although each $S$ and each $\Delta(S)$ are compact, if $\mathcal{S}$ is infinite, $\Omega$ and $\bigcup_{S \in \mathcal{S}} \Delta(S)$ are not compact.

\subsection{Marginals}

For a probability measure $\mu \in \Delta\left(S^{\prime}\right)$, the marginal $\mu_{\mid S}$ of $\mu$ on $S \preceq S^{\prime}$ is defined by

$$
\mu_{\mid S}(D):=\mu\left(\left(r_{S}^{S^{\prime}}\right)^{-1}(D)\right), \quad D \in \mathcal{F}_{S}
$$

Let $S_{\mu}$ be the space on which $\mu$ is a probability measure. Whenever $S_{\mu} \succeq S(E)$, then we abuse notation slightly and write

$$
\mu(E)=\mu\left(E \cap S_{\mu}\right) .
$$

If $S(E) \npreceq S_{\mu}$, then we say that $\mu(E)$ is undefined.

\subsection{Continuous type mappings}

Let $I$ be a nonempty finite or countable set of individuals. For every individual, each state gives rise to a probabilistic belief over states in some space.

Definition 1 For each individual $i \in I$, there is a continuous type mapping $t_{i}: \Omega \longrightarrow$ $\bigcup_{S \in \mathcal{S}} \Delta(S)$. We require the type mapping $t_{i}$ to satisfy the following properties: ${ }^{3}$

(0) Confinement: If $\omega \in S^{\prime}$ then $t_{i}(\omega) \in \Delta(S)$ for some $S \preceq S^{\prime}$.

(1) If $S^{\prime \prime} \succeq S^{\prime} \succeq S, \omega \in S^{\prime \prime}$, and $t_{i}(\omega) \in \triangle(S)$ then $t_{i}\left(\omega_{S^{\prime}}\right)=t_{i}(\omega)$.

(2) If $S^{\prime \prime} \succeq S^{\prime} \succeq S, \omega \in S^{\prime \prime}$, and $t_{i}(\omega) \in \triangle\left(S^{\prime}\right)$ then $t_{i}\left(\omega_{S}\right)=t_{i}(\omega)_{\mid S}$.

(3) If $S^{\prime \prime} \succeq S^{\prime} \succeq S, \omega \in S^{\prime \prime}$, and $t_{i}\left(\omega_{S^{\prime}}\right) \in \triangle(S)$ then $S_{t_{i}(\omega)} \succeq S$.

$t_{i}(\omega)$ represents individual $i$ 's belief at state $\omega$. Properties (0) to (3) guarantee the consistent fit of beliefs and awareness at different state-spaces. Confinement means that at any given state $\omega \in \Omega$, an individual's belief is concentrated on states that are all described with the same "vocabulary"- the "vocabulary" available to the individual at $\omega$. This "vocabulary" may be less expressive than the "vocabulary" used to describe

Footnote 2 continued

topology when $S$ is normal (and in particular compact and/or metric); the weakest topology for which the mapping

$$
\mu \longrightarrow \int_{S} f d \mu
$$

is continuous for every continuous real-valued function $f$ on $S$ (see Heifetz (2006), Fn. 3).

3 As is shown in Heifetz et al. (2013), Property (1) of the type mappings is implied by the Properties (0),(2), and (3). 
statements in the state $\omega$. ." Properties (1) to (3) compare the types of an individual in a state $\omega \in S^{\prime}$ and its projection to $\omega_{S}$, for some $S \preceq S^{\prime}$. Property (1) and (2) mean that at the projected state $\omega_{S}$ the individual believes everything she believes at $\omega$ given that she is aware of it at $\omega_{S}$. Property (3) means that at $\omega$ an individual cannot be unaware of an event that she is aware of at the projected state $\omega_{S^{\prime}}$.

Define $^{4}$

$$
\operatorname{Ben}_{i}(\omega):=\left\{\omega^{\prime} \in \Omega: t_{i}\left(\omega^{\prime}\right)_{\mid S_{t_{i}(\omega)}}=t_{i}(\omega)\right\}
$$

This is the set of states at which individual $i$ 's type or the marginal thereof coincides with her type at $\omega$. Such sets are events in our structure:

Remark 1 For any $\omega \in \Omega, \operatorname{Ben}_{i}(\omega)$ is a $S_{t_{i}(\omega)}$-based event, which is not necessarily measurable. 5

Assumption 1 If $\operatorname{Ben}_{i}(\omega) \subseteq E$, for a measurable event $E$, then $t_{i}(\omega)(E)=1$.

This assumption implies introspection (Property (va) in Proposition 4 in Heifetz et al. 2013). Note, that if $\operatorname{Ben}_{i}(\omega)$ is measurable, then Assumption 1 implies $t_{i}(\omega)\left(\operatorname{Ben}_{i}(\omega)\right)=1$.

Definition 2 We denote by $\underline{\Omega}:=\left\langle\mathcal{S},\left(r_{S^{\prime}}^{S^{\prime \prime}}\right)_{S^{\prime \prime} \succeq S^{\prime}},\left(t_{i}\right)_{i \in I}\right\rangle$ a topological unawareness belief structure.

Topological unawareness belief structures are analogous to unawareness belief structures in Heifetz et al. (2013) except for the additional topological properties and the well-foundedness asumption. See Heifetz et al. (2013) for further details and interpretations.

2.9 Belief, common certainty, and awareness

For $i \in I, p \in[0,1]$ and an event $E$, the $p$-belief operator is defined by

$$
B_{i}^{p}(E):=\left\{\omega \in \Omega: t_{i}(\omega)(E) \geq p\right\},
$$

if there is a state $\omega$ such that $t_{i}(\omega)(E) \geq p$, and by

$$
B_{i}^{p}(E):=\emptyset^{S(E)}
$$

otherwise. The mutual $p$-belief operator on events is defined by

$$
B^{p}(E)=\bigcap_{i \in I} B_{i}^{p}(E)
$$

\footnotetext{
4 The name "Ben" is chosen analogously to the "ken" in knowledge structures.

5 Even in a standard type space, if the $\sigma$-algebra is not countably generated, then the set of states where a player is of a certain type might not be measurable.
} 
The common certainty operator on events is defined by

$$
C B^{1}(E)=\bigcap_{n=1}^{\infty}\left(B^{1}\right)^{n}(E) .
$$

These are standard definitions (e.g. see Monderer and Samet 1989) adapted to our unawareness structures.

As in Heifetz et al. (2013), we define for every $i \in I$ the awareness operator

$$
A_{i}(E):=\left\{\omega \in \Omega: t_{i}(\omega) \in \Delta(S) \text { for some } S \succeq S(E)\right\},
$$

for every event $E$, if there is a state $\omega$ such that $t_{i}(\omega) \in \Delta(S)$ with $S \succeq S(E)$, and by

$$
A_{i}(E):=\emptyset^{S(E)}
$$

otherwise.

In Heifetz et al. (2013), Proposition 1 and 2, we show that $A_{i}(E), B_{i}^{p}(E), B^{p}(E)$, and $C B^{1}(E)$ are all $S(E)$-based measurable events. We also show in Heifetz et al. (2013), Proposition 4 that standard properties of belief obtain. Moreover, in Heifetz et al. (2013), Proposition 5, we show "standard" properties of awareness. One of those properties is weak necessitation, i.e., for any event $E \in \Sigma, A_{i}(E)=B_{i}^{1}\left(S(E)^{\uparrow}\right)$. This property will be used later in the proof.

Definition 3 An event $E$ is evident if for each $i \in I, E \subseteq B_{i}^{1}(E)$.

Proposition 1 For every event $F \in \Sigma$ :

(i) $C B^{1}(F)$ is evident, that is $C B^{1}(F) \subseteq B_{i}^{1}\left(C B^{1}(F)\right)$ for all $i \in I$.

(ii) There exists an evident event $E$ such that $\omega \in E$ and $E \subseteq B_{i}^{1}(F)$ for all $i \in I$, if and only if $\omega \in C B^{1}(F)$.

The proof is analogous to Proposition 3 in Monderer and Samet (1989) for a standard state-space and thus omitted.

\section{A generalized "No-speculative-trade" theorem}

Definition 4 (Prior) A prior for player $i$ is a system of probability measures $P_{i}=$ $\left(P_{i}^{S}\right)_{S \in \mathcal{S}} \in \prod_{S \in \mathcal{S}} \Delta(S)$ such that

1. The system is projective: If $S^{\prime} \preceq S$ then the marginal of $P_{i}^{S}$ on $S^{\prime}$ is $P_{i}^{S^{\prime}}$. (That is, if $E \in \Sigma$ is an event whose base-space $S(E)$ is lower or equal to $S^{\prime}$, then $P_{i}^{S}(E)=P_{i}^{S^{\prime}}(E)$.)

2. Each probability measure $P_{i}^{S}$ is a mixture of $i$ 's beliefs in $S$ : for every event $E \in \Sigma$ such that $S(E) \preceq S$,

$$
P_{i}^{S}\left(E \cap S \cap A_{i}(E)\right)=\int_{S \cap A_{i}(E)} t_{i}(\cdot)(E) d P_{i}^{S}(\cdot) .
$$


We call any probability measure $\mu_{i} \in \Delta(S)$ satisfying Eq. (1) in place of $P_{i}^{S}$ a prior of player $i$ on $S$.

Roughly the probability assigned by agent $i$ 's prior to the event $E \cap A_{i}(E)$ is the mixture of agent $i$ 's beliefs $t_{i}(\omega)(E)$ over states $\omega$ in which $i$ is aware of $E$ weighted by the prior. This is analogous to the definition of prior in standard belief structures (see Samet 1999). See Heifetz et al. (2013) for further discussions and examples.

Definition 5 (Common Prior) $P=\left(P^{S}\right)_{S \in \mathcal{S}} \in \prod_{S \in \mathcal{S}} \Delta(S)$ (resp. $P^{S} \in \Delta(S)$ ) is a common prior (resp. a common prior on $S$ ) if $P$ (resp. $P^{S}$ ) is a prior (resp. a prior on $S$ ) for every player $i \in I$.

Denote by $\left[t_{i}(\omega)\right]:=\left\{\omega^{\prime} \in \Omega: t_{i}\left(\omega^{\prime}\right)=t_{i}(\omega)\right\}$.

Definition 6 A common prior $P=\left(P^{S}\right)_{S \in \mathcal{S}} \in \prod_{S \in \mathcal{S}} \Delta(S)$ (resp. a common prior $P^{S}$ on $\left.S\right)$ is positive if and only if for all $i \in I$ and $\omega \in \Omega$ : if $t_{i}(\omega) \in \triangle\left(S^{\prime}\right)$, then $P^{S}\left(\left(\left[t_{i}(\omega)\right] \cap S^{\prime}\right)^{\uparrow} \cap S\right)>0$ for all $S \succeq S^{\prime}$ (resp. for $S$ ).

Note that with this positivity assumption, $\operatorname{Ben}_{i}(\omega)$ is measurable for every $\omega \in \Omega$ and $i$. Note further that by Lemma 3 below, $\left[t_{i}(\omega)\right] \cap S^{\prime} \in \mathcal{F}_{S^{\prime}}$.

Recall Remark 7 in Heifetz et al. (2013) according to which if $\hat{S}$ is the upmost statespace in the lattice $\mathcal{S}$, and $\left(P_{i}^{S}\right)_{S \in \mathcal{S}} \in \prod_{S \in \mathcal{S}} \Delta(S)$ is a tuple of probability measures, then $\left(P_{i}^{S}\right)_{S \in \mathcal{S}}$ is a prior for player $i$ if and only if $P_{i}^{\hat{S}}$ is a prior for player $i$ on $\hat{S}$ and $P_{i}^{S}$ is the marginal of $P_{i}^{\hat{S}}$ for every $S \in \mathcal{S}$.

Speculative trade between agents could occur at a state if it is common certainty that agents form different expectations of a random variable (e.g. stock returns).

Definition 7 Let $x_{1}$ and $x_{2}$ be real numbers and $v$ a continuous random variable ${ }^{6}$ on $\Omega$. Define the sets $E_{1}^{\leq x_{1}}:=\left\{\omega \in \Omega: \int_{S_{t_{1}(\omega)}} v(\cdot) d\left(t_{1}(\omega)\right)(\cdot) \leq x_{1}\right\}$ and $E_{2}^{\geq x_{2}}:=$ $\left\{\omega \in \Omega: \int_{S_{t_{2}(\omega)}} v(\cdot) d\left(t_{2}(\omega)\right)(\cdot) \geq x_{2}\right\}$. We say that at $\omega$, conditional on his information, player 1 (resp. player 2) believes that the expectation of $v$ is weakly below $x_{1}$ (resp. weakly above $x_{2}$ ) if and only if $\omega \in E_{1}^{\leq x_{1}}$ (resp. $\omega \in E_{1}^{\geq x_{2}}$ ).

Since we endowed $\Omega$ with the disjoint union topology, we have that a function $v: \Omega \longrightarrow \mathbb{R}$ is continuous if and only if for each $S \in \mathcal{S}$ the restriction of $v$ to $S$ is a continuous function from $S$ to $\mathbb{R}$.

\footnotetext{
${ }^{6}$ One may wonder to what extent the assumption of a continuous random variable would preclude situations in which for instance the "returns from investing in eurozone sovereign debt seems to dependent discontinuously on the question of whether the eurozone stays intact". We view continuity more as a technical assumption. The intuitive notion of continuity referred to in the eurozone example does not necessarily correspond to the mathematical notion of continuity. To see this, note that since we do not assume that state-spaces are connected, we allow for a states-space to be a disjoint union of two open subsets, call them $A$ and $B$, with $A$ standing for the event that the eurozone stays intact while $B$ corresponding to the event that the eurozone falls apart. Then, a random variable, which restricted to $\mathrm{A}$ is continuous and larger than $x$ while restricted to $B$ being also continuous but smaller than $y$ with $x>y$, is a continuous random variable although it depends "discontinuously" (i.e., more precisely it "jumps") on the event of whether or not the eurozone stays intact or not.
} 
Note that the sets $E_{1}^{\leq x_{1}}$ or $E_{1}^{\geq x_{2}}$ may not be events in our unawareness belief structure, because $v(\omega) \neq v\left(\omega_{S}\right)$ is allowed, for $\omega \in S^{\prime} \succ S$. Yet, we can define $p$-belief, mutual $p$-belief, and common certainty for measurable subsets of $\Omega$, and show that the properties stated in Heifetz et al. (2013), Propositions 4 and 6 obtain as well. The proofs are analogous and thus omitted.

Theorem 1 Let $\Omega$ be a topological unawareness belief structure and $P$ a positive common prior. Then, there is no state $\tilde{\omega} \in \Omega$, continuous random variable $v: \Omega \longrightarrow$ $\mathbb{R}$, and $x_{1}, x_{2} \in \mathbb{R}$ with $x_{1}<x_{2}$ such that: at $\tilde{\omega}$ it is common certainty that conditional on her information, player 1 believes that the expectation of $v$ is weakly below $x_{1}$ and, conditional on his information, player 2 believes that the expectation of $v$ is weakly above $x_{2}$.

This general "no-speculative-trade" theorem implies our "no-speculative-trade" theorem for finite unawareness belief structures (Heifetz et al. 2013).

We should clarify how the additional assumptions on the lattice and the statespaces allow us to generalize the "no-speculative-trade" theorem for finite unawareness belief structures in Heifetz et al. (2013) to the infinite case. One assumption imposed on topological unawareness structures is that the complete lattice of spaces is wellfounded. That is, we impose that each nonempty subset $\mathcal{X} \subseteq \mathcal{S}$ of the lattice contains at least one $\preceq$-minimal space. If the join is an element of the subset $\mathcal{X}$, then it is the unique $\preceq$-minimal space of $\mathcal{X}$. Otherwise well-foundedness implies that there must be several incomparable $\preceq$-minimal spaces. The assumption that the lattice of spaces is well-founded is used in an important step of the proof. If the set of states in which there is common certainty that the first player's expectation is strictly above $x$ and the second player's expectations is weakly below $x$ is nonempty, we need to find a minimal state-space such that the common certainty event restricted to this space is nonempty. Assuming that the lattice of spaces is well-founded allows us to find such a minimal space, which is key to extending the "no-speculative-trade" theorem to unawareness. Otherwise, it could be the case that agent 1 may believe that agent 2 is less aware and believes that agent 1 is even less aware and believes that agent 2 is even less aware and believes ...

The assumption that state-spaces are compact Hausdorff might not be strictly necessary. This is because our assumption of a positive common prior (Definition 6) implies that for each agent there can be at most a countable number of types. This fact should facilitate the development of results in a purely measure theoretic setting. Yet, our assumptions make the proofs of Lemmata 1, 2, and 3, and Theorem 1 simpler. Moreover, the topological unawareness belief structure could be of independent interest. For instance, it might prepare for other versions of the "no-speculative-trade" results, where the positivity assumption is dropped (and hence any player could have uncountably many types). Feinberg (2000) and Heifetz (2006) prove results on the absence of common certainty of speculative-trade in some states without the positivity assumption. We focus on the absence of common certainty of speculative-trade in all states. Hence, our notion of "no-speculative-trade" implies Feinberg's notion of "no-speculative-trade". Although this comes at the cost of the positivity assumption, we opted for our notion because intuitively one is interested to know whether there are some states (as opposed to all states) where agents speculate. 
On the feasibility of a converse to Theorem 1, we note that Heifetz et al. (2013) show by example that the converse of the "no-speculative-trade" theorem does not hold even in the finite case.

\section{Proof of Theorem 1}

\subsection{Preliminary definitions and results}

We define $G \subseteq \Omega$ to be a measurable set if and only if for all $S \in \mathcal{S}, G \cap S \in \mathcal{F}_{S}$. The collection of measurable sets forms a sigma-algebra on $\Omega$.

Let $\underline{\Omega}$ be an unawareness belief structure. As in Heifetz et al. (2012) Appendix B, we define the flattened type space associated with the unawareness belief structure $\underline{\Omega}$ by

$$
F(\underline{\Omega}):=\left\langle\Omega, \mathcal{F},\left(t_{i}^{F}\right)_{i \in I}\right\rangle
$$

where $\Omega$ is the union of all state-spaces in the unawareness belief structure $\Omega, \mathcal{F}$ is the collection of all measurable sets in $\underline{\Omega}$, and $t_{i}^{F}: \Omega \longrightarrow \Delta(\Omega)$ is defined by

$$
t_{i}^{F}(\omega)(E):= \begin{cases}t_{i}(\omega)\left(E \cap S_{t_{i}(\omega)}\right) & \text { if } E \cap S_{t_{i}(\omega)} \neq \emptyset \\ 0 & \text { otherwise }\end{cases}
$$

The definition of the belief operator as well as standard properties of belief and Proposition 1 can be extended to measurable subsets of $\Omega$. The proofs are analogous and thus omitted.

In this article, a standard topological type space (see for instance, Heifetz 2006) is a compact Hausdorff space $\Omega$ such that for every individual $i \in I$ there is a continuous type mapping $t_{i}: \Omega \longrightarrow \Delta(\Omega)$ from $\Omega$ to the the space of regular Borel probability measures $\Delta(\Omega)$ endowed with the topology of weak convergence.

Let $\Omega$ be a topological unawareness belief structure and $P$ a positive common prior. For the proof of the theorem, we have to show that there is no evident measurable set $E \in \mathcal{F}$ such that $\tilde{\omega} \in E$ and

$$
\int_{\Omega} v(\cdot) d\left(t_{1}(\omega)\right)(\cdot) \leq x_{1}<x_{2} \leq \int_{\Omega} v(\cdot) d\left(t_{2}(\omega)\right)(\cdot)
$$

for all $\omega \in E$.

We need the following lemmata:

Lemma 1 Let $\underline{\Omega}$ be a topological unawareness belief structure, $v: \Omega \longrightarrow \mathbb{R}$ be a continuous random variable, and $x \in \mathbb{R}$. Then, $\left\{\omega \in \Omega: \int_{\Omega} v(\cdot) d\left(t_{i}(\omega)\right)(\cdot) \geq x\right\}$ and $\left\{\omega \in \Omega: \int_{\Omega} v(\cdot) d\left(t_{i}(\omega)\right)(\cdot) \leq x\right\}$ are closed subsets of $\Omega .{ }^{7}$

\footnotetext{
${ }^{7}$ Note that we abuse notation and write $\int_{\Omega} v(\cdot) d\left(t_{i}(\omega)\right)(\cdot)$ instead of $\int_{S_{t_{i}}(\omega)} v(\cdot) d\left(t_{i}(\omega)\right)(\cdot)$.
} 
Proof of the Lemma Since for every $S \in \mathcal{S}$, the topology on $\Delta(S)$ coincides with the weak* topology and since in particular, $v: S \longrightarrow \mathbb{R}$ is continuous, $\left\{\mu \in \Delta(S): \int_{S} v(\cdot) d \mu(\cdot)<x\right\}$ is open in $\Delta(S)$. Hence, $\left\{v \in \bigcup_{S \in \mathcal{S}} \Delta(S): \int_{S} v(\cdot)\right.$ $d \nu(\cdot)<x\}$ is open in $\bigcup_{S \in \mathcal{S}} \Delta(S)$.

By the continuity of $t_{i}: \Omega \longrightarrow \bigcup_{S \in \mathcal{S}} \Delta(S)$, it follows that $\left\{\omega \in \Omega: \int_{\Omega} v(\cdot)\right.$ $\left.d\left(t_{i}(\omega)\right)(\cdot)<x\right\}$ is open in $\Omega$ and hence its relative complement with respect to $\Omega$, $\left\{\omega \in \Omega: \int_{\Omega} v(\cdot) d\left(t_{i}(\omega)\right)(\cdot) \geq x\right\}$ is closed in $\Omega$.

Lemma 2 Let $\Omega$ be a topological unawareness belief structure. Let $E$ be a closed subset of $\Omega$. Then, $C B^{1}(E)$ is a closed subset of $\Omega$.

Proof of the Lemma The relative complement of $E$ with respect to $\Omega, \Omega \backslash E$, is open, and hence for every $S \in \mathcal{S},(\Omega \backslash E) \cap S=S \backslash(E \cap S)$ is open in $S$. Therefore $\{\mu \in \Delta(S): \mu(S \backslash(E \cap S))>0\}$ is open. It follows that $\bigcup_{S \in \mathcal{S}}\{\mu \in \Delta(S)$ : $\mu(S \backslash(E \cap S))>0\}$ is open. Hence, for every $i \in I,\left\{\omega \in \Omega: t_{i}(\omega) \in \bigcup_{S \in \mathcal{S}}\{\mu \in \Delta(S)\right.$ : $\mu(S \backslash(E \cap S))>0\}\}$ is open. It follows that its relative complement with respect to $\Omega, B_{i}^{1}(E)=\left\{\omega \in \Omega: t_{i}(\omega) \in \bigcup_{S \in \mathcal{S}}\{\mu \in \Delta(S): \mu(E \cap S)=1\}\right\}$ is closed. Since an arbitrary intersection of closed sets is closed, the Lemma follows by induction.

Lemma 3 Let $\Omega$ be a topological unawareness belief structure. Then, for every $\omega \in \Omega$, every state-space $S \in \mathcal{S}$ and every player $i \in I$, the set $\left\{\omega^{\prime} \in \Omega: t_{i}\left(\omega^{\prime}\right)=t_{i}(\omega)\right\} \cap S$ is closed in $S$.

Proof of the Lemma Since $\Delta\left(S_{t_{i}(\omega)}\right)$ is the set of regular Borel probability measures on $S_{t_{i}(\omega)}$ endowed with the topology of weak convergence, $\left\{t_{i}(\omega)\right\}$ is closed in $\Delta\left(S_{t_{i}(\omega)}\right)$, and hence $\left\{t_{i}(\omega)\right\}$ is closed in $\bigcup_{S \in \mathcal{S}} \Delta(S)$. Therefore, by continuity of $t_{i}, t_{i}^{-1}\left(\left\{t_{i}(\omega)\right\}\right)=\left[t_{i}(\omega)\right]$ is closed in $\Omega$. Hence, $\left[t_{i}(\omega)\right] \cap S$ is closed in $S$.

Lemma 4 Let $\underline{\Omega}$ be a topological unawareness belief structure. Let $P^{S}$ be a positive (common) prior on the state-space $S$, and let $\omega \in S$ be such that $t_{i}(\omega) \in \Delta(S)$. Then, for every $E \in \mathcal{F}_{S}$, we do have $t_{i}(\omega)(E)=t_{i}(\omega)\left(E \cap\left[t_{i}(\omega)\right]\right)=\frac{P^{S}\left(E \cap\left[t_{i}(\omega)\right]\right)}{P^{S}\left(S \cap\left[t_{i}(\omega)\right]\right)}$.

Proof We have $t_{i}(\omega)\left(S \cap\left[t_{i}(\omega)\right]\right)=1$ and hence $t_{i}(\omega)(E)=t_{i}(\omega)\left(E \cap S \cap\left[t_{i}(\omega)\right]\right)=$ $t_{i}(\omega)\left(E \cap\left[t_{i}(\omega)\right]\right)$. Since $P^{S}$ is positive, we do have $P^{S}\left(S \cap\left[t_{i}(\omega)\right]\right)>0$.

Since $S\left(\left(E \cap\left[t_{i}(\omega)\right]\right)^{\uparrow}\right)=S$ and since $\omega^{\prime} \in\left[t_{i}(\omega)\right]$ implies $t_{i}\left(\omega^{\prime}\right) \in \Delta(S)$, we do have $\left(E \cap\left[t_{i}(\omega)\right]\right)^{\uparrow} \cap A_{i}\left(\left(E \cap\left[t_{i}(\omega)\right]\right)^{\uparrow}\right)=\left(E \cap\left[t_{i}(\omega)\right]\right)^{\uparrow}$. We also have $\left(S \cap\left[t_{i}(\omega)\right]\right)^{\uparrow} \subseteq A_{i}\left(S^{\uparrow}\right)=A_{i}\left(\left(E \cap\left[t_{i}(\omega)\right]\right)^{\uparrow}\right)$. The last equality follows from weak necessitation. We have-by the definition of a common prior-the following (with our abuse of notation):

$$
\begin{aligned}
P^{S}\left(E \cap\left[t_{i}(\omega)\right]\right)= & \int_{S \cap A_{i}\left(\left(E \cap\left[t_{i}(\omega)\right]\right)^{\uparrow}\right)} t_{i}(\cdot)\left(E \cap\left[t_{i}(\omega)\right]\right) d P^{S}(\cdot) \\
= & \int_{S \cap\left[t_{i}(\omega)\right]} t_{i}(\cdot)\left(E \cap\left[t_{i}(\omega)\right]\right) d P^{S}(\cdot) \\
& +\int_{\left(S \cap A_{i}\left(S^{\uparrow}\right)\right) \backslash\left(S \cap\left[t_{i}(\omega)\right]\right)} t_{i}(\cdot)\left(E \cap\left[t_{i}(\omega)\right]\right) d P^{S}(\cdot)
\end{aligned}
$$


But if $\omega^{\prime} \in\left(S \cap A_{i}\left(\left(E \cap\left[t_{i}(\omega)\right]\right)^{\uparrow}\right)\right) \backslash\left(S \cap\left[t_{i}(\omega)\right]\right)$, then $t_{i}\left(\omega^{\prime}\right)\left(E \cap\left[t_{i}(\omega)\right]\right)=0$, and hence, we have

$$
\begin{aligned}
P^{S}\left(E \cap\left[t_{i}(\omega)\right]\right) & =\int_{S \cap\left[t_{i}(\omega)\right]} t_{i}(\cdot)\left(E \cap\left[t_{i}(\omega)\right]\right) d P^{S}(\cdot) \\
& =t_{i}(\omega)\left(E \cap\left[t_{i}(\omega)\right]\right) \int_{S \cap\left[t_{i}(\omega)\right]} 1 d P^{S}(\cdot) \\
& =t_{i}(\omega)\left(E \cap\left[t_{i}(\omega)\right]\right) P^{S}\left(S \cap\left[t_{i}(\omega)\right]\right) .
\end{aligned}
$$

Since $P^{S}\left(S \cap\left[t_{i}(\omega)\right]\right)>0$, it follows that $t_{i}(\omega)\left(E \cap\left[t_{i}(\omega)\right]\right)=\frac{P^{S}\left(E \cap\left[t_{i}(\omega)\right]\right)}{P^{S}\left(S \cap\left[t_{i}(\omega)\right]\right)}$.

\subsection{Proof of the Theorem}

Suppose by contradiction that there are $x_{1}, x_{2} \in \mathbb{R}$ with $x_{1}<x_{2}$ and a continuous random variable $v: \Omega \longrightarrow \mathbb{R}$ such that $C B^{1}\left(E_{1}^{\leq x_{1}} \cap E_{2}^{\geq x_{2}}\right) \neq \emptyset$, where

$$
\begin{aligned}
& E_{1}^{\leq x_{1}}:=\left\{\omega \in \Omega: \int_{S_{t_{1}(\omega)}} v(\cdot) d\left(t_{1}(\omega)\right)(\cdot) \leq x_{1}\right\}, \text { and } \\
& E_{2}^{\geq x_{2}}:=\left\{\omega \in \Omega: \int_{S_{t_{2}(\omega)}} v(\cdot) d\left(t_{2}(\omega)\right)(\cdot) \geq x_{2}\right\} .
\end{aligned}
$$

Let $S$ be a $\preceq$-minimal state-space with the property that $S \cap C B^{1}\left(E_{1}^{\leq x_{1}} \cap E_{2}^{\geq x_{2}}\right) \neq \emptyset$.

By standard properties of beliefs, we have $C B^{1}\left(E_{1}^{\leq x_{1}} \cap E_{2}^{\geq x_{2}}\right) \subseteq B_{i}^{1}\left(C B^{1}\left(E_{1}^{\leq x_{1}} \cap\right.\right.$ $\left.E_{2}^{\geq x_{2}}\right)$ ) for $i=1,2$. This implies that for each $\omega \in S \cap C B^{1}\left(E_{1}^{\leq x_{1}} \cap E_{2}^{\geq x_{2}}\right)$ and $i=1,2$, we have $t_{i}(\omega)\left(C B^{1}\left(E_{1}^{\leq x_{1}} \cap E_{2}^{\geq x_{2}}\right)\right)=1$, which by the minimality of $S$ implies that $t_{i}(\omega) \in \Delta(S)$ and $t_{i}(\omega)\left(S \cap C B^{1}\left(E_{1}^{\leq x_{1}} \cap E_{2}^{\geq x_{2}}\right)\right)=1$.

By Lemma 2, $S \cap C B^{1}\left(E_{1}^{\leq x_{1}} \cap E_{2}^{\geq x_{2}}\right)$ is closed in $S$. Therefore it is easy to verify that if flattened, $F\left(S \cap C B^{1}\left(E_{1}^{\leq x_{1}} \cap E_{2}^{\geq x_{2}}\right)\right)$, that is $S \cap C B^{1}\left(E_{1}^{\leq x_{1}} \cap E_{2}^{\geq x_{2}}\right)$ with the induced structure, is a standard topological type space (as in Heifetz 2006), since for each $\omega \in S \cap C B^{1}\left(E_{1}^{\leq x_{1}} \cap E_{2}^{\geq x_{2}}\right)$, we have $t_{i}(\omega)\left(S \cap C B^{1}\left(E_{1}^{\leq x_{1}} \cap E_{2}^{\geq x_{2}}\right)\right)=1$ for $i=1,2$.

Since $P^{S}$ is a positive prior on $S$, we have that $P^{S}\left(S \cap\left[t_{i}(\omega)\right]\right)>0$, for each $\omega \in S$.

For $\omega \in S \cap C B^{1}\left(E_{1}^{\leq x_{1}} \cap E_{2}^{\geq x_{2}}\right)$, we also have $t_{i}(\omega)\left(S \cap C B^{1}\left(E_{1}^{\leq x_{1}} \cap E_{2}^{\geq x_{2}}\right) \cap\right.$ $\left.\left[t_{i}(\omega)\right]\right)=1$, and by Lemma 4 , we have $t_{i}(\omega)\left(S \cap C B^{1}\left(E_{1}^{\leq x_{1}} \cap E_{2}^{\geq x_{2}}\right) \cap\left[t_{i}(\omega)\right]\right)=$ $\frac{P^{S}\left(S \cap C B^{1}\left(E_{1}^{\leq x_{1}} \cap E_{2}^{\geq x_{2}}\right) \cap\left[t_{i}(\omega)\right]\right)}{P^{S}\left(S \cap\left[t_{i}(\omega)\right]\right)}$.

Hence, since $P^{S}\left(S \cap\left[t_{i}(\omega)\right]\right)>0$, it follows that $P^{S}\left(S \cap C B^{1}\left(E_{1}^{\leq x_{1}} \cap E_{2}^{\geq x_{2}}\right) \cap\right.$ $\left.\left[t_{i}(\omega)\right]\right)=P^{S}\left(S \cap\left[t_{i}(\omega)\right]\right)>0$. It follows that $P^{S}\left(S \cap C B^{1}\left(E_{1}^{\leq x_{1}} \cap E_{2}^{\geq x_{2}}\right)\right)>$ 
0 . Therefore, it is easy to check that $\frac{P^{S}(\cdot)}{P^{S}\left(S \cap C B^{1}\left(E_{1}^{\leq x_{1}} \cap E_{2}^{\geq x_{2}}\right)\right)}$ is a common prior on $F\left(S \cap C B^{1}\left(E_{1}^{\leq x_{1}} \cap E_{2}^{\geq x_{2}}\right)\right)$.

Claim Let $\omega \in C B^{1}\left(E_{1}^{\leq x_{1}} \cap E_{2}^{\geq x_{2}}\right) \cap S$. Then, $\int_{S \cap C B^{1}\left(E_{1}^{\leq x_{1}} \cap E_{2}^{\geq x_{2}}\right)} v(\cdot) d\left(t_{1}(\omega)\right)(\cdot) \leq$ $x_{1}$ and $\int_{S \cap C B^{1}\left(E_{1}^{\leq x_{1}} \cap E_{2}^{\geq x_{2}}\right)} v(\cdot) d\left(t_{2}(\omega)\right)(\cdot) \geq x_{2}$.

We prove the second inequality, the first is analogous to the second one. We know already that $t_{2}(\omega) \in \Delta(S)$. By the definitions, $\omega \in S \cap C B^{1}\left(E_{1}^{\leq x_{1}} \cap E_{2}^{\geq x_{2}}\right)$ implies $\omega \in S \cap B_{2}^{1}\left(E_{2}^{\geq x_{2}}\right)$, and therefore $t_{2}(\omega)\left(\left[t_{2}(\omega)\right] \cap E_{2}^{\geq x_{2}} \cap S\right)=1$. It follows that $\left[t_{2}(\omega)\right] \cap$ $E_{2}^{\geq x_{2}} \cap S$ is nonempty. Let $\omega^{\prime} \in\left[t_{2}(\omega)\right] \cap E_{2}^{\geq x_{2}} \cap S$. Then, we have $\int_{S} v(\cdot) d\left(t_{2}\left(\omega^{\prime}\right)\right)(\cdot) \geq$ $x_{2}$. But we have $t_{2}(\omega)=t_{2}\left(\omega^{\prime}\right)$ and therefore $\int_{S} v(\cdot) d\left(t_{2}(\omega)\right)(\cdot) \geq x_{2}$.

Since $S$ is compact and $v: S \longrightarrow \mathbb{R}$ is continuous, there is a $\bar{v} \in \mathbb{R}$ such that $|v(\tilde{\omega})| \leq \bar{v}$ for all $\tilde{\omega} \in S$.

Since $t_{2}(\omega)\left(S \cap C B^{1}\left(E_{1}^{\leq x_{1}} \cap E_{2}^{\geq x_{2}}\right)\right)=1$, we have

$$
\begin{aligned}
\left|\int_{S \backslash\left(S \cap C B^{1}\left(E_{1}^{\leq x_{1}} \cap E_{2}^{\geq x_{2}}\right)\right)} v(\cdot) d\left(t_{2}(\omega)\right)(\cdot)\right| & \leq \bar{v} \int_{S \backslash\left(S \cap C B^{1}\left(E_{1}^{\leq x_{1}} \cap E_{2}^{\geq x_{2}}\right)\right)} 1 d\left(t_{2}(\omega)\right)(\cdot) \\
& =\bar{v} \quad t_{2}(\omega)\left(S \backslash\left(S \cap C B^{1}\left(E_{1}^{\leq x_{1}} \cap E_{2}^{\geq x_{2}}\right)\right)\right) \\
& =0 .
\end{aligned}
$$

Hence, we have

$$
\int_{S \cap C B^{1}\left(E_{1}^{\leq x_{1}} \cap E_{2}^{\geq x_{2}}\right)} v(\cdot) d\left(t_{2}(\omega)\right)(\cdot)=\int_{S} v(\cdot) d\left(t_{2}(\omega)\right)(\cdot) \geq x_{2}
$$

and this finishes the proof of the claim.

It follows that we have found a standard topological type space $S \cap C B^{1}\left(E_{1}^{\leq x_{1}} \cap\right.$ $E_{2}^{\geq x_{2}}$ ) in the sense of Heifetz (2006) with a common prior and a continuous random variable $v: S \cap C B^{1}\left(E_{1}^{\leq x_{1}} \cap E_{2}^{\geq x_{2}}\right) \longrightarrow \mathbb{R}$ such that

$$
\int_{S \cap C B^{1}\left(E_{1}^{\leq x_{1}} \cap E_{2}^{\geq x_{2}}\right)} v(\cdot) d\left(t_{1}(\omega)\right)(\cdot) \leq x_{1}<x_{2} \leq \int_{S \cap C B^{1}\left(E_{1}^{\leq x_{1}} \cap E_{2}^{\geq x_{2}}\right)} v(\cdot) d\left(t_{2}(\omega)\right)(\cdot) .
$$

Note that if we replace $v(\cdot)$ by $v(\cdot)-\frac{x_{1}+x_{2}}{2}$, we get

$$
\begin{aligned}
& \int_{S \cap C B^{1}\left(E_{1}^{\leq x_{1}} \cap E_{2}^{\geq x_{2}}\right)} v(\cdot)-\frac{x_{1}+x_{2}}{2} d\left(t_{1}(\omega)\right)(\cdot)<0<\int_{S \cap C B^{1}\left(E_{1}^{\leq x_{1}} \cap E_{2}^{\geq x_{2}}\right)} v(\cdot) \\
& -\frac{x_{1}+x_{2}}{2} d\left(t_{2}(\omega)\right)(\cdot) .
\end{aligned}
$$


But this is a contradiction to Feinberg (2000) Theorem (Proposition 1 in Heifetz 2006). Hence, this completes the proof of the theorem.

Acknowledgments We thank an anonymous reviewer for helpful comments. We also thank Aviad Heifetz for helpful discussions. Martin acknowledges financial support from the Spanish Ministerio de Educación y Ciencia via a Ramon y Cajal Fellowship and Research Grant SEJ2004-07861, and the Fundación Ramón Areces. Burkhard received financial support through National Science Foundation award SES-0647811.

\section{References}

Billingsley, P.: Convergence of probability measures, John Wiley \& Sons (1968)

Board, O., Chung, K.S.: Object-based unawareness: theory and applications, mimeo (2011)

Feinberg, Y.: Characterizing common priors in form of posteriors. J. Econ. Theory 91, 127-179 (2000)

Grant, S., Quiggin, J.: Inductive reasoning about unawareness. Econ. Theory 54, 717-755 (2013)

Heifetz, A.: The positive foundation of the common prior assumption. Games Econ. Behav. 56, 105-120 (2006)

Heifetz, A., Meier, M., Schipper, B.C.: Unawareness, beliefs, and speculative trade. Games Econ. Behav. 77, 100-121 (2013)

Heifetz, A., Meier, M., Schipper, B.C. Unawareness, beliefs, and speculative trade, mimeo., University of California, Davis (2012)

Heifetz, A., Meier, M., Schipper, B.C.: A canonical model for interactive unawareness. Games Econ Behav 62, 304-324 (2008)

Heifetz, A., Meier, M., Schipper, B.C.: Interactive unawareness. J. Econ. Theory 130, 78-94 (2006)

Milgrom, P., Stokey, N.: Information, trade and common knowledge. J. Econ. Theory 26, 17-27 (1982)

Monderer, D., Samet, D.: Approximating common knowledge with common beliefs. Games Econ. Behav. 1, 170-190 (1989)

Samet, D.: Bayesianism without learning. Res. Econ. 53, 227-242 (1999) 